\title{
Henoch-Schönlein purpura: a rare vasculitis in older adults
}

\author{
${ }^{1}$ VK Daripally, ${ }^{2} \mathrm{NS}$ Shah \\ ${ }^{1}$ Specialty Trainee; ${ }^{2}$ Consultant Geriatrician; Department of Geriatric Medicine, University Hospitals of Leicester NHS Trust, \\ Leicester Royal Infirmary, UK
}

ABSTRACT Henoch-Schönlein purpura (HSP) is a small vessel vasculitis with immunoglobulin A-dominant immune deposits found in vessel walls. It primarily affects children, is uncommon in adults and often under-recognised in the elderly population due to its rarity in this group. When a purpuric rash develops in an older person it is typically treated with antibiotics for presumed infection, rather than investigated as potentially HSP. It is important to recognise this condition, particulary

Correspondence to $\mathbf{N}$ Shah, Department of Geriatric Medicine, Leicester Royal Infirmary, Leicester LEI 5WW, UK in older adults, as diagnosis has implications for management and prognosis. We tel. $+44(0) 1162585057$ e-mail Nainal.Shah@uhl-tr.nhs.uk report a case of vasculitis with renal involvement in an older person.

KEYWORDS Henoch-Schönlein purpura, vasculitis, older people

DECLARATION OF INTERESTS No conflicts of interest declared.

\section{CASE REPORT}

A 76-year-old woman presented with a three-day history of rash on her legs and lower back and she reported feeling tired. She had no history of arthralgia, or any other relevant symptoms. Two weeks before presentation she had been to the Isle of Wight in the UK, when she developed generalised aches and diarrhoea which settled spontaneously within a few days. Five days before presentation she was given a course of clarithromycin for a presumed chest infection. The antibiotic was stopped after two days by her general practitioner as she developed a rash. Past medical history included controlled hypertension, hypothyroidism, herpes zoster, plantar fasciitis and irritable bowel syndrome, with no previous history of rash. She was currently taking perindopril, bendroflumethiazide, simvastatin, levothyroxine and mebeverine. On examination a palpable, purpuric rash was visible over her feet, legs, buttocks and lower back (Figure I). Her temperature was normal and her blood pressure was $130 / 94 \mathrm{~mm} \mathrm{Hg}$. Systemic examination found nothing else remarkable. Initial investigations revealed a serum urea level of II.8 millimoles per litre (mmol/L) (normal range 2.5-6.5 $\mathrm{mmol} / \mathrm{L}$ ) and a creatinine level of 155 micromoles per litre $(\mu \mathrm{mol} / \mathrm{L})$ (normal range 60-120 $\mu \mathrm{mol} / \mathrm{L}$ ). Previous renal electrolytes were documented as within normal range. The haemoglobin level was 12.4 grams per deciliter $(\mathrm{g} / \mathrm{dL})$ (normal range $13-18 \mathrm{~g} / \mathrm{dL}$ ), the platelet count was $515 \times 10^{9}$ per litre (normal range $140-400 \times$ $10^{\circ} / \mathrm{L}$ ) and the International Normalised Ratio (INR) was I.3 (normal range 0.9-I.2). Routine urinalysis revealed haematuria and trace proteinuria.

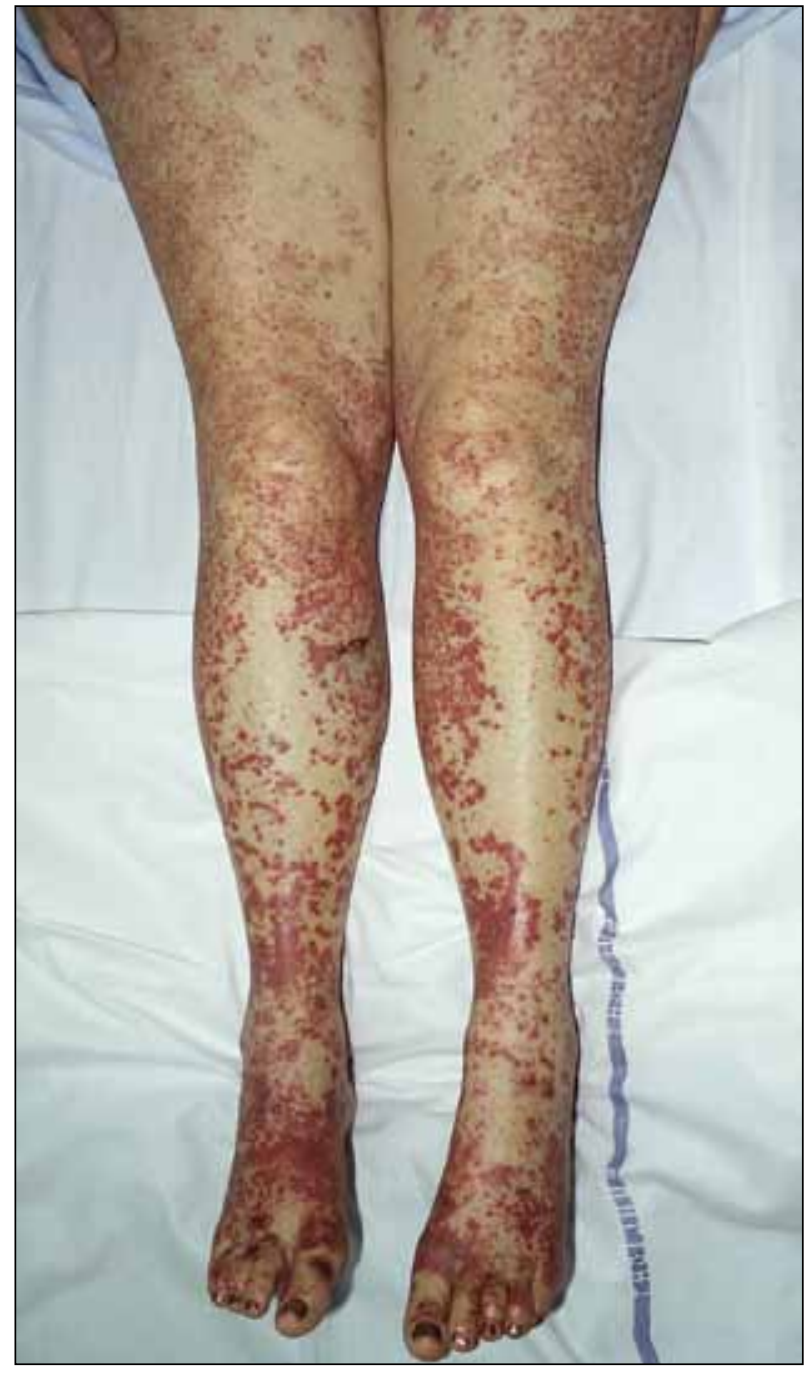

FIGURE I Vasculitic rash over the front of both legs. 
The patient was admitted due, in particular, to the vasculitic rash and apparent acute kidney injury. Blood cultures did not grow any organisms. Several tests were carried out: autoantibody screen, a rheumatoid factor test, hepatitis B and C tests, a test for anti-glomerular basement membrane antibodies, a myeloma screen, a glandular fever screen, an antistreptolysin $O$ titre test and a cytomegalovirus serology test, and they were all negative. Immunoglobulins and complement levels were within normal limits. Cryoglobulins were not detected. Epstein-Barr virus (EBV) immunoglobulin G (lgG) was detected which indicated past EBV exposure. A skin biopsy revealed granular immunoglobulin A2 (IgA2) deposits in the superficial dermal blood vessel walls and perivascular deposits of fibrin, findings consistent with Henoch-Schönlein purpura (HSP) (Figures 2 and 3). A renal ultrasound showed no gross structural abnormality.

The patient's renal function and rash improved spontaneously following a conservative treatment of rest, analgesia and adequate renal hydration. In view of the acute kidney injury at presentation, a nephrologist's opinion was requested and it was determined that a renal biopsy was not needed as renal function had improved. She was discharged home and followed up as an outpatient at four, eight and 12 weeks and at six months and one year. On follow-up visits her urinanalysis, blood pressure and kidney function remained stable.

\section{DISCUSSION}

Henoch-Schönlein purpura is the most common form of systemic vasculitis in young people, with an annual incidence of approximately 20 per 100,000 children under 17-years-old (with a peak incidence among children aged between four and six years).' The condition is identified by its clinical mani-festations - palpable purpura without thrombocytopaenia or coagulation abnormalities, arthralgia, abdominal pain and renal impairment or injury. It is uncommon in adults and often under-recognised in the elderly. The pathophysiology and natural history of HSP is not well described in adults, unlike in children, where extensive studies have been published. $^{2}$

Henoch-Schönlein purpura is an immune-mediated vasculitis involving small vessels, with the deposition of immune complexes containing $\lg A$ within affected organs. Skin biopsies of purpuric lesions demonstrate the involvement of small vessels (primarily postcapillary venules) within the papillary dermis. The predominant cell types within the inflammatory infiltrate are neutrophils and monocytes. Immunofluorescence studies show deposition of IgA, fibrin and the complement component $\mathrm{C} 3$ within the affected vessel walls. HenochSchönlein purpura is thought to be related to concomitant infection (upper respiratory tract infection, group $A$ streptococcus, varicella, hepatitis $B, E B V$ and mycoplasma

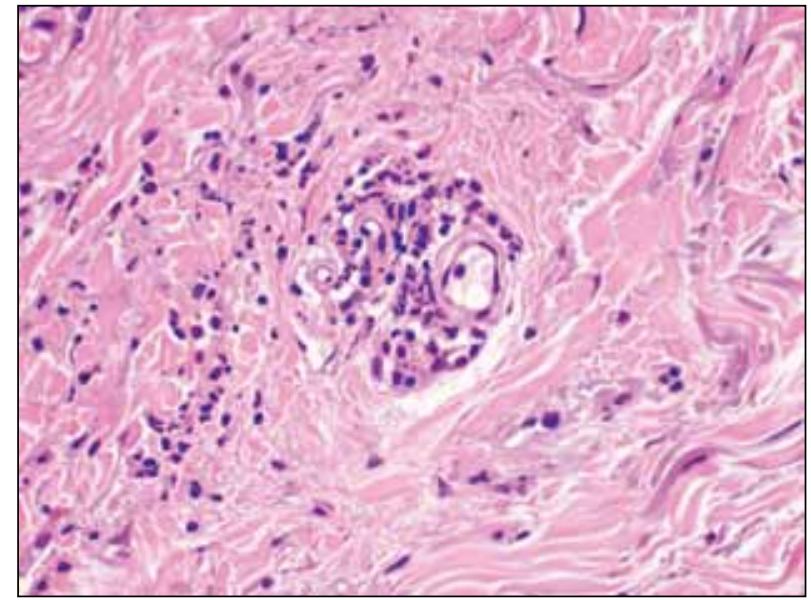

FIGURE 2 Capillaries with surrounding leucocytoclasis.

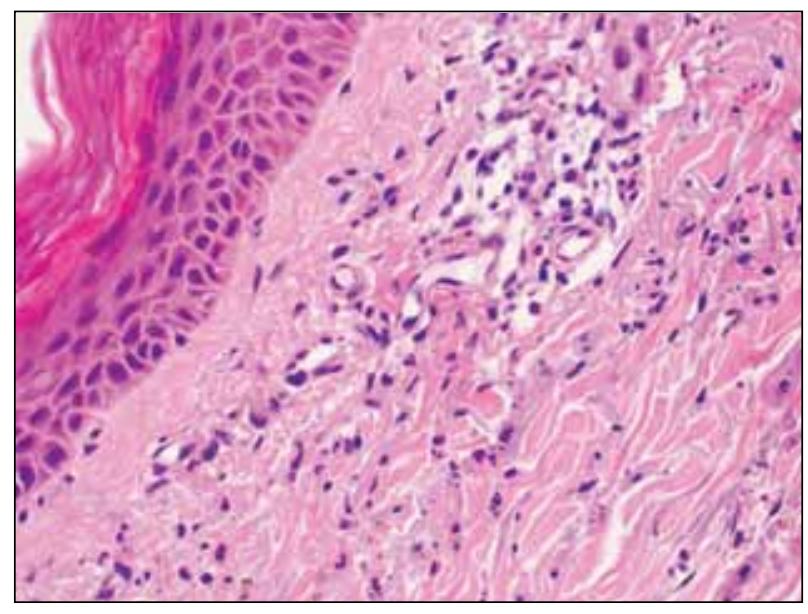

FIGURE 2 Capillaries with surrounding leucocytoclasis and fibrin.

have all been associated with HSP). Some drugs (penicillin, erythromycin, quinines, chlorpromazine), neoplasms (leukaemia, lymphoma, breast, lung, kidney, prostate, colon, cervix cancers and melanoma) and several miscellaneous conditions (such as pregnancy, familial Mediterranean fever and cryoglobulinemia) can also trigger HSP. A combination of genetic predisposition, environmental and immunological factors play a role in the pathogenesis of HSP.

\section{MANIFESTATIONS AND SYMPTOMS}

The classic clinical manifestations of HSP may develop over days and/or weeks and may vary in their order of presentation. The vasculitic rash typically appears in crops, is symmetrically distributed and located primarily in the buttocks and lower extremities. Arthritis/ arthralgia can occur in about two-thirds of patients. ${ }^{2}$ The arthritis is usually transient or migratory, typically oligoarticular, and non-deforming. Large joints are a common symptom in children, while smaller joints are found in adults presenting with the disease. Gastrointestinal manifestations of HSP in adults may be 
severe and include generalised abdominal pain, vomiting, gastrointestinal bleeding, diarrhoea and intestinal obstruction. ${ }^{3}$ HSP with renal involvement tends to be more severe in adults (with an incidence of about 63 per cent compared to 33 per cent in children with $\mathrm{HSP}^{4}$ ) with an increased risk of progression to end-stage renal disease. ${ }^{2.5}$ It may manifest as isolated haematuria and/or proteinuria, renal hypertension, nephritic syndrome and severe acute renal failure. The central nervous system can also be affected by HSP and the most common symptoms are headache, behavioural changes and seizures. Other rare manifestations include conduction abnormalities, congestive heart failure, acute myocardial infarction and pulmonary haemorrhage (more common in older patients). ${ }^{6}$

\section{DIAGNOSIS}

A variety of classification criteria for HSP have been proposed, but these are primarily used in research and have not been validated for use in the diagnosis of individual cases. In 2005, consensus criteria were developed by the Paediatric Rheumatology European Society (PRES) and the European League against Rheumatism (EULAR). ${ }^{7}$ These standards are more appropriate for diagnosing the illness in paediatric settings. The diagnosis of HSP is based on classic symptoms and signs, the most common of which is palpable purpura of the lower extremities and buttocks. In adult patients, diagnosis should be confirmed by biopsy, as HSP is rare in this group. Biopsies of the skin are usually adequate for diagnosing HSP as they will show leukocytoclastic vasculitis with a predominance of IgA deposition. A renal biopsy is reserved for patients with severe renal impairment or if diagnosis is uncertain.

Differential diagnosis of HSP in adults includes other systemic autoimmune diseases that can produce similar symptoms, such as hypersensitivity vasculitis, other small vessel vasculitides such as microscopic polyangiitis, Churg-Strauss syndrome, Wegener's disease, cryoglobulinemia and vascular inflammation secondary to a connective tissue disorder (e.g. systemic lupus erythematosus, rheumatoid arthritis, Behcet's disease and relapsing polychondritis) or to an infectious disease (e.g. hepatitis B or C).

Our patient showed the classic purpuric rash and also had renal involvement. The diagnosis was confirmed by a positive skin biopsy showing IgA immune complexes which differentiated this case from other small vessel vasculitides. The likely cause of HSP in this patient was possibly an antecedent respiratory tract infection (this was not confirmed) or the recent use of a macrolide antibiotic.

\section{TREATMENT}

Management of adults with HSP is primarily supportive therapy with rest, analgesia and adequate renal hydration. Glucocorticosteroid use may improve abdominal symptoms in adults with symptomatically severe GI involvement ${ }^{3}$ and may enhance the rate of resolution of the arthritis, however this treatment does not prevent recurrence of the disease. ${ }^{8}$ Treatment of the renal disease should only be considered in patients with marked proteinuria and/or impaired renal function during the acute episode. A renal biopsy is recommended in this circumstance. There is no evidence that conventional doses of steroids has a beneficial effect in patients with renal involvement. In patients with advanced disease however, a regimen consisting of highdose pulsed intravenous methylprednisolone (250 to $1000 \mathrm{mg}$ per day for three days) followed by oral prednisone for three months may be beneficial. ${ }^{910}$ Most patients with renal disease show a complete recovery (up to 94 per cent in children and 89 per cent in adults) at a mean follow-up of approximately 18 months. ${ }^{4}$ Recurrence (relapse) of HSP is common, occurring in up to one-third of patients and may be more likely among patients with renal involvement. ${ }^{7}$ Among adults, persistent proteinuria, hypertension and renal insufficiency are much more common and reported rates of end-stage renal disease range from 10 to 30 per cent at 15 years. . $^{211-14}$

Routine follow-up of all patients with HSP is recommended, with blood pressure checks and urinalysis monitoring weekly or biweekly for the first one to two months after presentation. Once the disease appears to be diminishing, monitoring should be monthly or every other month. If the urine analysis remains normal after the first six months, no further follow-up is required. ${ }^{15}$ If not, follow-up tests should continue for a year following initial presentation. Extended follow-up is often necessary however, particularly in older adults. Patients with persistent proteinuria, hypertension, or renal impairment should be referred to a nephrologist for further evaluation and treatment. Elderly patients with unexplained HSP should be screened for occult neoplasm and in patients with malignancy, they should be screened for metastatic disease. ${ }^{16}$

\section{CONCLUSION}

Henoch-Schönlein purpura is usually self-limiting, in contrast to other forms of systemic vasculitis. The majority of patients have mild symptoms and signs, need only supportive measures and may be cared for in an ambulatory setting. Short-term morbidity in HSP patients is primarily a result of gastrointestinal complications and long-term morbidity is usually a result of renal disease. Recurrence of symptoms can occur in up to one-third of patients. More aggressive treatment and extended 
follow-up with repeated urine analysis are often necessary to improve the outcome, especially in older patients. ${ }^{17}$ HSP should be considered as a potential diagnosis in adults and the elderly presenting with skin vasculitis, ${ }^{18}$ in order to prevent long-term morbidity.

\section{REFERENCES}

I Gardner-Medwin JM, Dolezalova P, Cummins C et al. Incidence of Henoch-Schönlein purpura, Kawasaki disease, and rare vasculitides in children of different ethnic origins. Lancet 2002; 360: I 197-202. http://dx.doi.org/I0.10I6/S0|40-6736(02) I I279-7

2 Pillebout E, Thervet E, Hill G et al. Henoch-Schönlein purpura in adults: outcome and prognostic factors. J Am Soc Nephrol 2002; | 3: | 27|-8. http://dx.doi.org//0.1097/0I.ASN.00000 I3883.99976.22

3 Trouillier S, Andre M, Delevaux I et al. [Abdominal manifestations of Henoch-Schönlein purpura in adults. A retrospective study of 23 cases.] Rev Med Interne 2009; 30:66I-70. http://dx.doi. org/I0.1016/j.revmed.2009.02.026

4 Rieu P, Noel LH. Henoch-Schönlein nephritis in children and adults. Morphological features and clinicopathological correlations. Ann Med Interne (Paris) 1999; I50: I5I-9.

5 Blanco R, Martínez-Taboada VM, Rodríguez-Valverde $\mathrm{V}$ et al. Henoch-Schönlein purpura in adulthood and childhood: two different expressions of the same syndrome. Arthritis Rheum 1997; 40:859-64. http://dx.doi.org/10.1002/art. 17804005I3

6 Usui K, Ochiai T, Muto R et al. Diffuse pulmonary hemorrhage as a fatal complication of Schönlein-Henoch purpura. J Dermatol 2007; 34:705-8. http://dx.doi.org/I0.I I I I/j.1346-8I38.2007.00364.x

7 Ozen S, Ruperto N, Dillon MJ et al. EULAR/PReS endorsed consensus criteria for the classification of childhood vasculitides. Ann Rheum Dis 2006; 65:936-4I. http://dx.doi.org/I0.II36/ ard.2005.046300

8 Saulsbury FT. Henoch-Schönlein purpura in children. Report of 100 patients and review of the literature. Medicine (Baltimore) 1999 78:395-409. http://dx.doi.org/I0.1097/00005792-1999/ I000-00005

9 Niaudet P, Habib R. Methylprednisolone pulse therapy in the treatment of severe forms of Schönlein-Henoch purpura nephritis. Pediatr Nephrol 1998; 12:238-43. http://dx.doi.org//0.1007/ s004670050446

\section{Acknowledgements}

We would like to thank Dr Gerald Saldanha MRCP(UK), FRCPath, PhD Department of Cancer Studies \& Molecular Medicine, University of Leicester, for help with the histopathology slides.

10 Balow JE. Renal vasculitis. Kidney Int 1985; 27:954-64. http://dx.doi. org/l 0.1038/ki.1985.104

I I Kellerman PS. Henoch-Schönlein purpura in adults. Am J Kidney Dis 2006; 48: 1009-16. http://dx.doi.org/10.1053/j.ajkd.2006.08.03।

12 Coppo R, Andrulli S, Amore A et al. Predictors of outcome in Henoch-Schönlein nephritis in children and adults. Am J Kidney Dis 2006; 47:993-1003. http://dx.doi.org/10.1053/j.ajkd.2006.02.178

13 Shrestha S, Sumingan N, Tan J et al. Henoch-Schönlein purpura with nephritis in adults: adverse prognostic indicators in a UK population. QJM 2006; 99:253-65. Erratum in: QJM 2006; 99:493. http://dx.doi.org/I0.1093/qjmed/hcl034

I4 Fogazzi GB, Pasquali S, Moriggi M et al. Long-term outcome of Schönlein-Henoch nephritis in the adult. Clin Nephrol 1989; 31:60-6.

15 Narchi H. Risk of long term renal impairment and duration of follow up recommended for Henoch-Schönlein purpura with normal or minimal urinary findings: a systematic review. Arch Dis Child 2005; 90:916-20. http://dx.doi.org/I0.II36/adc.2005.07464 I

16 Zurada JM, Ward KM, Grossman ME. Henoch-Schönlein purpura associated with malignancy in adults. J Am Acad Dermatol 2006; 55:S65-70. http://dx.doi.org/I0.1016/j.jaad.2005.10.0I I

17 Hung SP, Yang YH, Lin YT et al. Clinical manifestations and outcomes of Henoch-Schönlein purpura: comparison between adults and children. Pediatr Neonatol 2009; 50:162-8. http://dx.doi. org/I0.I0I6/SI875-9572(09)60056-5

18 Diehl MP, Harrington T, Olenginski T. Elderly-onset Henoch-Schönlein purpura: a case series and review of the literature. J Am Geriatr Soc 2008; 56:2 I57-9. http://dx.doi.org/I 0.I I I I/j.I532-54I5.2008.0I983.x 